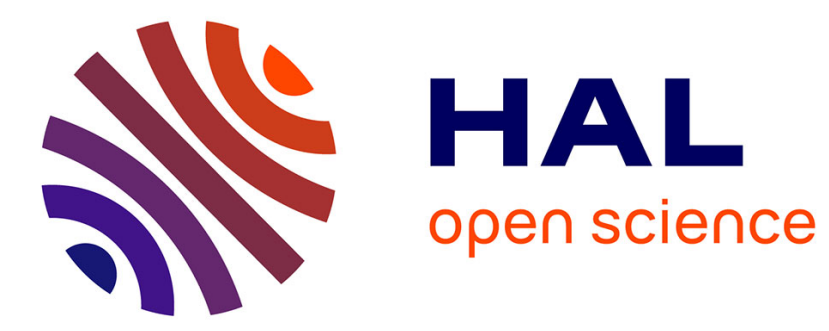

\title{
Unimodular-Upper polynomial matrix decomposition for MIMO Spatial Multiplexing
}

\author{
Moustapha Mbaye, Moussa Diallo, Mamadou Mboup
}

\section{To cite this version:}

Moustapha Mbaye, Moussa Diallo, Mamadou Mboup. Unimodular-Upper polynomial matrix decomposition for MIMO Spatial Multiplexing. 15th IEEE International Workshop on Signal Processing Advances in Wireless Communications (SPAWC14), Jun 2014, Toronto, Canada. hal-00995923

HAL Id: hal-00995923

https://hal.inria.fr/hal-00995923

Submitted on 25 May 2014

HAL is a multi-disciplinary open access archive for the deposit and dissemination of scientific research documents, whether they are published or not. The documents may come from teaching and research institutions in France or abroad, or from public or private research centers.
L'archive ouverte pluridisciplinaire HAL, est destinée au dépôt et à la diffusion de documents scientifiques de niveau recherche, publiés ou non, émanant des établissements d'enseignement et de recherche français ou étrangers, des laboratoires publics ou privés. 


\section{Unimodular-Upper polynomial matrix decomposition for MIMO Spatial Multiplexing}

\author{
Moustapha Mbaye \\ moustapha.mbaye.sn@gmail.com \\ University Cheikh Anta Diop \\ BP 5005 Dakar Fann, Sénégal
}

\author{
Moussa Diallo \\ moussa.diallo@ucad.edu.sn \\ University Cheikh Anta Diop \\ BP 5005 Dakar Fann, Sénégal
}

\author{
Mamadou Mboup \\ Mamadou.Mboup@univ-reims.fr \\ CReSTIC Université de Reims Champagne Ardenne \\ BP 1039 Moulin de la Housse, 51687 Reims cedex 2 France
}

\begin{abstract}
We present a simple algorithm to compute the factors of a Unimodular-Upper (UU) polynomial matrix decomposition. The algorithm relies on the classical $L U$ factorization and the inverse of the unimodular factor is also provided. Such decomposition is useful for spatial multiplexing in MIMO channel transmission system since it enables to reduce the MIMO channel matrix into independent SISO channels by a pre- and post-filtering. Unlike the classical QR-based polynomial matrix Singular Values Decomposition (QR-PMSVD), the proposed UU method allows to completely cancel the co-channel interference (CCI). Moreover, most of the resulting independent SISO channels are likely to be reduced to simple additive noise channels, i.e. with no InterSymbol Interference. However, the noise is coloured and possibly enhanced due to the non unitary property of the corresponding post filter. The complexity and sum rate capacity performance of the proposed method are studied and compared with QR-PMSVD.
\end{abstract}

\section{INTRODUCTION}

Let $H(z) \in \mathbb{C}^{p \times q}$ a polynomial matrix, i.e.

$$
H(z)=\sum_{k=0}^{L} H_{k} z^{k}
$$

where each coefficient $H_{k}$ is a $p \times q$-complex matrix and $L$ is the formal degree of the polynomial.

We consider the problem of the decomposition of $H(z)$ in the form

$$
H(z)=U(z) D(z) V(z),
$$

where the factors $V(z), D(z)$ and $U(z)$ are simple and structured polynomial matrices. The difficulty lies in the constraint that the factors be also polynomial. Such decomposition is useful in Multiple-Input Multiple-Output (MIMO) channel transmission system. The polynomial matrix $H(z)$ then represents the FIR channel transfer function.

The inverse of the factors $U(z)$ and $V(z)$ are used as postand pre-coders respectively in order to reduce the MIMO channel to a simpler form $D(z)$. These inverses are not only assumed to exist but also, it is required that they are Laurent polynomials and easy, if not immediate, to compute.

Ideally, the form of $D(z)$ is diagonal [1], [5], [7]. Diagonalization of the system in space means the transformation of the MIMO channel in $N=\min (p, q)$ separate and independant single input single output (SISO) channels, so that there is no co-channel interference (CCI). This is achieved for example when a singular value decomposition (SVD) is considered. This decomposition assumes that $V(z)$ and $U(z)$ are (FIR) paraunitary polynomial matrices, i.e. they satisfy

$$
\widetilde{U}(z) U(z)=I \text { and } V(z) \widetilde{V}(z)=I, \quad \text { for all } z \in \mathbb{C},
$$

where the tilde stands for the paraconjugation, defined by: $\widetilde{X}(z)=X^{*}(1 / \bar{z})$. For instance, the paraconjugate of $X(z)=$ $\sum_{k} X_{k} z^{k}$ is given by $\widetilde{X}(z)=\sum_{k} X_{k}^{*} z^{-k}$. The sign $*$ denotes the transpose-conjugate.

Note however that a polynomial matrix SVD with polynomial factors does not exist in general [4]. Two main approaches are found in the literature:

Forster, McWhirter et al. [3]:

Apply successive elementary Laurent polynomial Givens rotations to iteratively zero the largest coefficient in magnitude, beneath the diagonal. This produces FIR paraunitary factors $U(z)$ and $V(z)$. The factor $D(z)$ is also a Laurent polynomial but only approximately diagonal: the largest coefficients in magnitude, beneath the diagonal are less than a prescribed tolerance parameter $\epsilon$. The effect of $\epsilon$ in the CCI as well as in the system performance is studied in [7]. In addition, the degrees of the involved polynomials grow fast in general, this is the reason why a truncation step is introduced. However, the paraunitary property is lost with the truncation, which degree is measured by some parameter $\mu$.

D. Cescato and H. Bolcskei [2]:

Apply classical SVD decomposition to samples of the original matrix $H\left(z_{i}\right)=U\left(z_{i}\right) D\left(z_{i}\right) V\left(z_{i}\right), i=1,2, \ldots$. The factors of the polynomial decomposition are deduced by polynomial interpolation of the samples of the factors respectively. However the nice properties of the sample matrices (easy invertibility, paraunitary,...) are not guaranteed to hold after interpolation.

In this paper, we investigate a Unimodular-Upper (UU) decomposition for polynomial matrices, from the classical $L U$ decomposition. More precisely, given a $\mathbb{C}^{p \times q}$ polynomial matrix $H(z)$, the problem is to write it in the form

$$
H(z)=U(z) R(z)
$$

where $R(z)$ is an upper triangular polynomial matrix and $U(z)$ is a unimodular polynomial matrix. Recall that a unimodular matrix is a square polynomial matrix with constant nonzero 
determinant. It therefore admits a polynomial inverse. Applying the same decomposition to the transpose of the resulting factor $R(z)$ above provides a factorization of the form:

$$
H(z)=U(z) D(z) V(z),
$$

where $V(z)$ and $U(z)$ are two unimodular matrices and $D(z)$ is a diagonal polynomial matrix. The rest of this paper is organized as follow. The proposed UU decomposition is detailed in section II. It is well known that such decomposition always exists and can be obtained from solutions of Bezout equations. However, we present a simple and explicit algorithm to compute the factor $D(z)$ and the unimodular matrices $U(z)$ and $V(z)$ together with their respective inverses. Then section III is devoted to the calculation of the sum rate. In the section IV, some numerical results regarding the sum rates attained by the different transmission schemes and their cost in complexity is presented.

\section{UU POLYNOMIAL MATRIX DECOMPOSITION}

The proposed UU decomposition follows the same steps as the classical LU factorization (or Gauss elimination). In each step, however, a preprocessing is required in order to reduce the pivot element to a constant, subsequently set to 1 . To begin, let us take a simple example of a $4 \times 4$ polynomial matrix.

$$
H(z)=\left[\begin{array}{llll}
h_{1,1}(z) & h_{1,2}(z) & h_{1,3}(z) & h_{1,4}(z) \\
h_{2,1}(z) & h_{2,2}(z) & h_{2,3}(z) & h_{2,4}(z) \\
h_{3,1}(z) & h_{3,2}(z) & h_{3,3}(z) & h_{3,4}(z) \\
h_{4,1}(z) & h_{4,2}(z) & h_{4,3}(z) & h_{4,4}(z)
\end{array}\right]
$$

Assume that the polynomials $h_{1,1}(z)$ and $h_{2,1}(z)$ are coprime. Then, let us denote by $\left[h_{1,1}^{\sharp}(z), h_{2,1}^{\sharp}(z)\right]$ any pair of polynomials solving the Bezout equation.

$$
h_{1,1}^{\sharp}(z) h_{1,1}(z)+h_{2,1}^{\sharp}(z) h_{2,1}(z)=1 .
$$

If necessary, we may consider a permutation of the rows of $H(z)$ in order to get (2). This may however be insufficient, e.g. when the first column of $H(z)$, noted $\boldsymbol{h}_{1}(z)$, is not an irreducible polynomial vector. In such case we may write:

$\boldsymbol{h}_{1}(z)=d(z)\left[\hat{h}_{1,1}(z) \hat{h}_{2,1}(z) \hat{h}_{3,1}(z) \hat{h}_{4,1}(z)\right]^{t} \triangleq d(z) \widehat{\boldsymbol{h}}_{1}(z)$ where $d(z)$ is the greatest commom divisor $(g c d)$ of the components of $\boldsymbol{h}_{1}(z)$. The remaining polynomial vector $\widehat{\boldsymbol{h}}_{1}(z)$ is thus irreducible and we assume that the Bezout equation (2) holds by replacing the polynomials $h_{i, 1}(z)$ by their reduced counterparts $\hat{h}_{i, 1}(z)$. In this latter cased we set $d_{1}(z)=d(z)$. Otherwise, $d_{1}(z)=1$ and $\widehat{\boldsymbol{h}}_{1}(z)=\boldsymbol{h}_{1}(z)$. If, nonetheless (2) still fails for all pairs while $\boldsymbol{h}_{1}(z)$ is irreducible, then the right hand side of (2) is replaced by the $g c d$ of the considered pair and the algorithm proceeds, following the procedure above.

Next, we introduce the block diagonal matrix

$$
\bar{A}_{1}(z)=\left[\begin{array}{cc|c|c}
h_{1,1}^{\sharp}(z) & h_{2,1}^{\sharp}(z) & & \\
-h_{2,1}(z) & h_{1,1}(z) & & \\
\hline & & 1 & 1
\end{array}\right]=\left[\begin{array}{cc|c}
\bar{B}_{1}(z) & 0 \\
\hline 0 & I_{2}
\end{array}\right]
$$

where $I_{k}$ is the $k \times k$ identity matrix. A direct verification shows that

$$
\widetilde{H}_{1}(z)=\bar{A}_{1}(z) H(z)=\left[\begin{array}{cccc}
d_{1}(z) & \widetilde{h}_{1,2}(z) & \widetilde{h}_{1,3}(z) & \widetilde{h}_{1,4}(z) \\
0 & \widetilde{h}_{2,2}(z) & \widetilde{h}_{2,3}(z) & \widetilde{h}_{2,4}(z) \\
h_{3,1}(z) & h_{3,2}(z) & h_{3,3}(z) & h_{3,4}(z) \\
h_{4,1}(z) & h_{4,2}(z) & h_{4,3}(z) & h_{4,4}(z)
\end{array}\right]
$$

Now, one can apply a first step of a classical Gaussian elimination to zero the off-diagonal elements of the first column. This amounts to multiplying the above matrix by the lower triangular polynomial matrix:

$$
\bar{L}_{1}(z)=\left[\begin{array}{c|ccc}
1 & & & \\
\hline 0 & 1 & & \\
-\hat{h}_{3,1}(z) & & 1 & \\
-\hat{h}_{4,1}(z) & & & 1
\end{array}\right]
$$

The result is denoted by $H_{1}(z)=\bar{L}_{1}(z) \bar{A}_{1}(z) H(z)$ and it reads as:

$$
H_{1}(z)=\left[\begin{array}{c|ccc}
d_{1}(z) & \widetilde{h}_{1,2}(z) & \widetilde{h}_{1,3}(z) & \widetilde{h}_{1,4}(z) \\
\hline 0 & \widetilde{h}_{2,2}(z) & \widetilde{h}_{2,3}(z) & \widetilde{h}_{2,4}(z) \\
0 & \widetilde{h}_{3,2}(z) & \widetilde{h}_{3,3}(z) & \widetilde{h}_{3,4}(z) \\
0 & \widetilde{h}_{4,2}(z) & \widetilde{h}_{4,3}(z) & \widetilde{h}_{4,4}(z)
\end{array}\right]
$$

The same steps are now repeated for the lower-right block of $H_{1}(z)$. In the second column we obtain:

$$
H_{2}(z)=\bar{L}_{2}(z) \bar{A}_{2}(z) H_{1}(z)
$$

Finally, we obtain the triangular polynomial matrix

$$
R(z)=\bar{A}_{3}(z) H_{2}(z)=\left[\begin{array}{cccc}
d_{1}(z) & \widetilde{h}_{1,2}(z) & \widetilde{h}_{1,3}(z) & \widetilde{h}_{1,4}(z) \\
0 & d_{2}(z) & \check{h}_{2,3}(z) & \check{h}_{2,4}(z) \\
0 & 0 & d_{3}(z) & \hat{h}_{3,4}(z) \\
0 & 0 & 0 & \hat{h}_{4,4}(z)
\end{array}\right]
$$

To summarize, note that this last matrix reads as

$$
R(z)=\bar{A}_{3}(z) \bar{L}_{2}(z) \bar{A}_{2}(z) \bar{L}_{1}(z) \bar{A}_{1}(z) H(z)
$$

Each matrix $\bar{L}_{i}(z)$ is lower triangular unimodular and its inverse is obtained by simply changing the sign of the offdiagonal entries. Each $\bar{B}_{i}(z)$ as in (3) is also unimodular and:

$$
-J \bar{B}_{i}^{t}(z) J=\bar{B}_{i}^{-1}(z) \text {, where } J=\left[\begin{array}{rr}
0 & 1 \\
-1 & 0
\end{array}\right], i=1,2,3 \text {. }
$$

Now on, we set: $\quad A_{i}(z)=\bar{A}_{i}(z)^{-1}, L_{i}(z)=\bar{L}_{i}(z)^{-1}$.

Then, the preceding steps provide a UU decompostion of the polynomial matrix $H(z)$ :

$$
H(z)=U(z) R(z)
$$

where $U(z)=A_{1}(z) L_{1}(z) A_{2}(z) L_{2}(z) A_{3}(z)$ is a unimodular polynomial matrix and $R(z)$ is the upper triangular polynomial matrix obtained in the last step of the algorithm described above. Applying the same decomposition to the right factor in (9), namely $R(z)^{t}=V(z)^{t} D(z)$ provides a factorization of the form $H(z)=U(z) D(z) V(z)$, where $V(z)$ and $U(z)$ are two unimodular matrices and $D(z)$ is a diagonal polynomial matrix. 
This decomposition can be generalized for any given matrix $H \in \mathbb{C}^{p \times q}$. In the general case, the decomposition ends up after a number of iteration given by $\min (p, q)$. The factors $V^{-1}(z)$ and $U^{-1}(z)$ can be used as post- and pre-coders respectively in order to reduce the MIMO channel to its equivalent $D(z)$. Since $D(z)$ is exactly diagonal, there is no co-channel interference (CCI) unlike with other methods [3], [2]. Note also that we may verify by direct inspection that, for exemple, the first entry of $D(z)$ will correspond to the $\mathrm{gcd}$ of all the polynomials in the first row and first column of $H(z)$. This shows that the probability that the diagonal elements of $D(z)$ be equal to one is high, except for the last one.

\section{SUM RATE CAPACITY}

Let $H(z) \in \mathbb{C}^{p \times q}$ a wideband MIMO channel and $U_{p o}(z)$ and $V_{p r}(z)$ the post- and pre-coders respectively. The communication process in spatial multiplexing context is:

$$
y=U_{p o}(z) H(z) V_{p r}(z) x(z)+U_{p o}(z) n(z)
$$

where $y \in \mathbb{C}^{p \times 1}$ is the received signal, $x \in \mathbb{C}^{q \times 1}$ the signal vector with covariance $\mathrm{E}\left(x x^{*}\right)=\sigma_{x}^{2} I_{q}$ and $n$ a white Gaussian noise vector with covariance $\mathrm{E}\left(n n^{*}\right)=\sigma_{n}^{2} I_{p}$

By introducing the decomposition of $H(z)$, (10) becomes:

$$
y=U_{p o}(z) U(z) D(z) V(z) V_{p r}(z) x(z)+U_{p o}(z) n(z)
$$

The reduced MIMO channel depends on the decomposition and we consider the following two possibilities:

- UU decompostion with $U_{p o}=U^{-1}$ and $V_{p r}=V^{-1}$ : In this case, $y=D(z) x(z)+U^{-1}(z) n(z)$. There is no CCI because $D(z)$ is diagonal. However $U(z)$ is not paraunitary and this will affect the filtered noise properties. By considering an equal gain transmission scheme [6], the sum rate can be simply calculated by:

$$
\sum_{k=1}^{\min (p, q)} \int_{0}^{2 \pi} \log _{2}\left|1+\frac{\sigma_{x}^{2} D_{k, k}\left(e^{\jmath \omega}\right) D_{k, k}^{*}\left(e^{\jmath \omega}\right)}{\sigma_{n}^{2} U_{k,:}^{-1}\left(e^{\jmath \omega}\right)\left(U_{k,:}^{-1}\right)^{*}\left(e^{\jmath \omega}\right)}\right| d \omega
$$

where $D_{k, k}(\cdot)$ are the diagonal elements of $D(\cdot), U_{k, \text { : }}$ stands for the $k$-th line of $U$.

- QR-PMSVD of Forster, McWhirter et al. [3]: In this case, $D(z)$ is only approximately diagonal. Equation (11) becomes:

$$
y=\underbrace{\widetilde{U}(z) U(z)}_{I_{N_{r}}+U_{e}} \underbrace{D(z)}_{\Delta+D_{e}} \underbrace{V(z) \tilde{V}(z)}_{I_{N_{t}}+V_{e}} x(z)+\underbrace{\widetilde{U}(z) n(z)}_{w}
$$

where $U_{e}$ and $V_{e}$ are the errors caused by the truncation and $D_{e}$ is the off diagonal part of $D(z)$ caused by the fact that the convergence criterion $\epsilon \neq 0$. Thereby (13) can be rewritten as:

$$
y=\Delta(z) x(z)+C(z) x(z)+w(z)
$$

where $C(z)$ represents the CCI filter and $w(z)$ the noise component. Since $\Delta(z)$ is diagonal, the sum rate can be calculated by:

$$
\sum_{k=1}^{\min (p, q)} \int_{0}^{2 \pi} \log _{2}\left|1+\frac{\sigma_{x}^{2} \Delta_{k, k}\left(e^{\jmath \omega}\right) \Delta_{k, k}^{*}\left(e^{\jmath \omega}\right)}{\sigma_{x}^{2} \Phi\left(e^{\jmath \omega}\right)+\sigma_{n}^{2} U_{k,:}^{*}\left(e^{\jmath \omega}\right) U_{k,:}\left(e^{\jmath \omega}\right)}\right| d \omega .
$$

where we have set $\Phi\left(e^{\jmath \omega}\right)=C_{k,:}^{*}\left(e^{\jmath \omega}\right) C_{k,:}\left(e^{\jmath \omega}\right)$.

\section{Simulations Results}

For the numerical simulations, we consider a spatial multiplexing scheme with $N_{t}$ transmit antennas and $N_{r}$ receive antennas. The MIMO channel $H(z)$ is modeled as:

$$
H(z)=\sum_{l=0}^{L-1} H_{l} e^{-\alpha l} z^{l}
$$

where the elements of the $H_{l}$ 's are obtained from a zeromean circularly symmetric normalized Gaussian distribution and $\alpha \in \mathbb{R}^{+}$is added in order to obtain an exponentially decaying power-delay-profile.

The figures 1 and 2 show the impulse responses of the diagonalized matrix $D(z)$ obtained respectively by QR-PMSVD and UU decomposition.

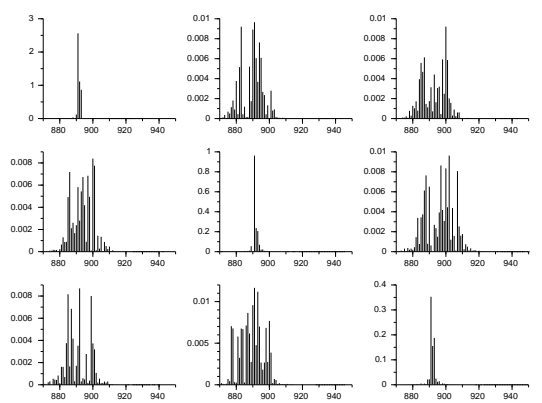

Fig. 1. Impulse response of diagonalized Matrix $D(z)$ obtained by QRPMSVD decomposition with $\epsilon=0.01$ and $\mu=10^{-6}$.

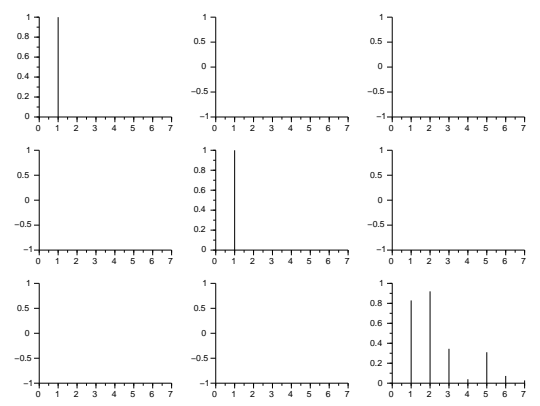

Fig. 2. Impulse response of diagonalized Matrix $D(z)$ obtained by UU decomposition.

The matrix $D(z)$ is not diagonal with QR-PMSVD algorithm. However, the coefficients beneath the diagonal are in magnitude less than $\epsilon$ which is set to 0.01 in this simulation. Clearly, the decomposition error decreases for decreasing $\epsilon$. By contrast, the complexity for post- and pre-coding may increase significantly, as shown in Table I which illustrates the maximum length of the post- and pre-coders. Unlike QRPMSVD, $D(z)$ is diagonal with UU and the post- and precoder are much less complex (see Table I).

The comparison in terms of sum rate versus SNR obtained through a MIMO $2 \times 2$ and MIMO $3 \times 3$ schemes, with several values of QR-PMSVD algorithm parameters, is represented respectively in Fig. 3 and Fig. 4. 
TABLE I

MAXIMUM LENGTH (NUMBER OF COEFFICIENTS) OF THE POST-CODER AND PRE-CODER FIR

\begin{tabular}{|c|c|c|c|c|}
\hline \multirow{2}{*}{ Parameters } & \multicolumn{2}{|c|}{ MIMO 2x2 } & \multicolumn{2}{c|}{ MIMO 3x3 } \\
\cline { 2 - 5 } & Pre-coder & Post-coder & Pre-coder & Post-coder \\
\hline UU & 11 & 6 & 28 & 12 \\
\hline \hline QR-PMSVD $\epsilon=10^{-1} \mu=10^{-3}$ & 28 & 44 & 81 & 121 \\
\hline QR-PMSVD $\epsilon=10^{-1} \mu=10^{-6}$ & 28 & 44 & 81 & 121 \\
\hline QR-PMSVD $\epsilon=10^{-2} \mu=10^{-3}$ & 442 & 447 & 821 & 1056 \\
\hline QR-PMSVD $\epsilon=10^{-2} \mu=10^{-6}$ & 622 & 628 & 954 & 1279 \\
\hline QR-PMSVD $\epsilon=10^{-3} \mu=10^{-3}$ & 746 & 835 & 1589 & 1751 \\
\hline QR-PMSVD $\epsilon=10^{-3} \mu=10^{-6}$ & 1127 & 1247 & 2041 & 2164 \\
\hline
\end{tabular}

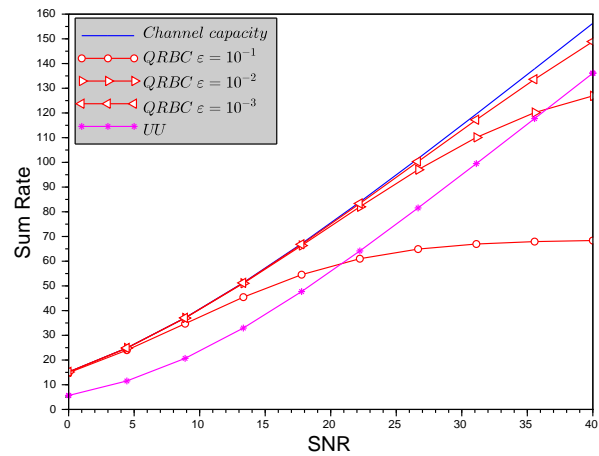

Fig. 3. Sum Rate averaged over 100 MIMO $2 \times 2$ Channel realization for UU and QR-PMSVD decomposition. The Channel length was $L=7$, the truncation parameter $\mu=10^{-6}$ for various $\epsilon$.

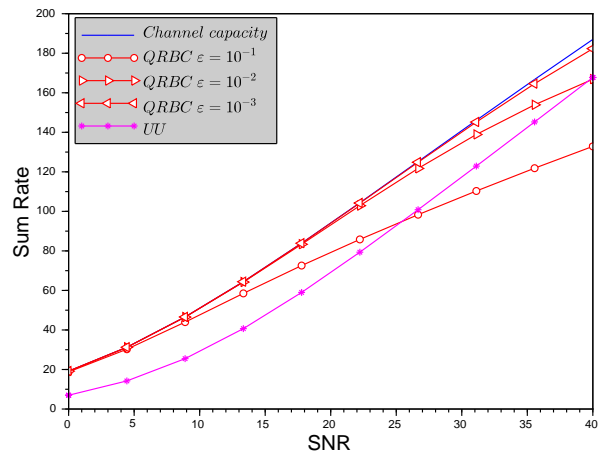

Fig. 4. Sum Rate averaged over 100 MIMO $3 \times 3$ Channel realization for UU and QR-PMSVD decomposition. The Channel length was $L=7$, the truncation parameter $\mu=10^{-6}$ for various $\epsilon$.

Firstly, the sum rate obtained with UU algorithm can not achieve the channel capacity. This is due to the fact that the post-coder $\left(U^{-1}\right)$ is not para-unitary and then the noise component is affected (see (12)).

Secondly, in the QR-PMSVD case, the sum rate is almost equal to the channel's capacity when $\epsilon$ is selected close to zero (here $\epsilon=10^{-3}$ ). However, if this parameter increases for obtaining more realistic FIR post- and pre-coder, as close to that obtained with UU (see Table.I), the sum rate with QRPMSVD becomes worse due to the important CCI.

To finish, we analyze the elements in $D(z)$ obtained here by UU decomposition. All diagonal elements of $D(z)$ are equal to 1 , except the last one. Thus, the first $N_{G}=\min \left(N_{t}, N_{r}\right)-1$ separate SISO channels are equivalent to Gaussian channel. Hence, there is no inter-symbol interference (ISI) on these channels and the equalization becomes very simple. Figure 5, which presents the received signal constellation with $4-Q A M$ modulation in MIMO $3 \times 3$ context, shows the effect of ISI. As we can see in figure boxes UU-antenna 1 and UU-antenna 2 , which represent the first $N_{G}=2$ SISO channels, there is no ISI. We just have the noise component effect. However in the other figure boxes we note the presence of ISI. As we have previously noted, this situation of no ISI happens in general with high probability.

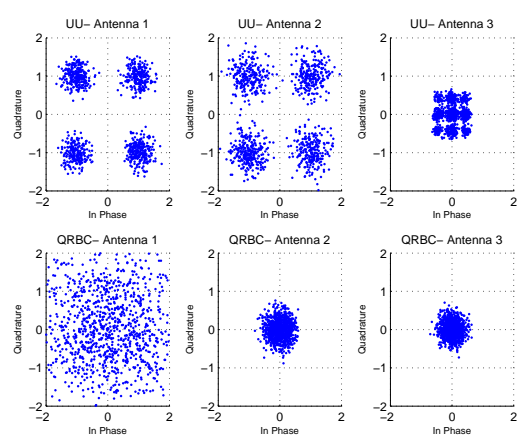

Fig. 5. Received signal constellation for 4-QAM modulation in MIMO $3 \times 3$ context. The SNR is set to $30 d B$.

\section{CONCLusion}

The proposed UU polynomial decomposition algorithm completely eliminates the CCI in MIMO spatial multiplexing scheme. In addition, except in the last SISO channel, the ISI in all channels is likely to be absent. The sum rate obtained with the decomposition can not, however, achieve the channel capacity because of the non unitary effect of the post-coder. Nevertheless, with equal complexity, the obtained sum rate is better than that achieved by the classical QR-based polynomial matrix approximate SVD decomposition.

\section{REFERENCES}

[1] Rasmus Brandt. Polynomial matrix decompositions: Evaluation of algorithms with an application to wideband MIMO communications. MSc thesis, School of Electrical Engineering, Uppsala University, Stockholm, Sweden, 2010.

[2] D. Cescato and H. Bolcskei. QR decomposition of Laurent polynomial matrices sampled on the unit circle. IEEE Transactions on Information Theory, 56(9):4754-4761, Sept 2010.

[3] J. A. Foster, J.G. McWhirter, M. R. Davies, and J.A. Chambers. An algorithm for calculating the QR and Singular Value Decompositions of polynomial matrices. IEEE Transactions on Signal Processing, 58(3):1263-1274, March 2010.

[4] S. Icart and P. Comon. Some properties of Laurent polynomial matrices. In 9th IMA Intern. Conf. on Math. in Sig. Proc., Birmingham, 2012.

[5] S. M. Razavi and T. Ratnarajah. Subspace beamforming via block SVD for MIMO system. In IEEE Sig. Proc. Adv. in Wireless Comm. (SPAWC), 2012.

[6] Shang-Ho Tsai. An equal gain transmission in MIMO wireless communications. In Global Telecommunications Conference (GLOBECOM 2010), 2010 IEEE, pages 1-5, 2010.

[7] H. Zamiri-Jafarian and M. Rajabzadeh. A polynomial matrix SVD approach for time domain broadband beamforming in MIMO-OFDM systems. In IEEE Conference Vehicular Technology, 2008. 